\title{
Feasibility Study of Holography Using Microwave Scattering*)
}

\author{
Hayato TSUCHIYA, Naofumi IWAMA, Soichiro YAMAGUCHI ${ }^{1)}$, \\ Ryota TAKENAKA ${ }^{2)}$ and Mayuko KOGA ${ }^{2)}$ \\ National Institute for Fusion Science, Toki 509-5292, Japan \\ ${ }^{1)}$ Kansai University, Suita 564-8680, Japan \\ ${ }^{2)}$ University of Hyogo, Himeji 671-2280, Japan
}

(Received 26 December 2018 / Accepted 17 July 2019)

\begin{abstract}
Microwave, unlike visible light, can be measured directly on the phase of the wave. The measurement of complex amplitude suggests a possibility of holographic plasma imaging with a single view-field of planar array of detectors. In this paper, an inverse problem of holography is formulated with respect to reflection and scattering wave observations. Against the restricted view-field and few detectors, a solution of Tikhonov type is proposed and examined by numerical simulations. The first result of feasibility study is presented.
\end{abstract}

(C) 2019 The Japan Society of Plasma Science and Nuclear Fusion Research

Keywords: microwave diagnostics, inverse problem, complex amplitude, holography

DOI: $10.1585 /$ prr.14.3402146

\section{Introduction}

In the magnetized plasma confinement study, the methods of the good confinement mode (H-mode) and the detached plasma mode have been discovered, and the physics descriptions of them are being understood. In particular, the plasma turbulence is understood to be important for the transport and influential also to the whole plasma profile via the nonlinear coupling among multi-scale phenomena. In the experimental study, however, the diagnostics for turbulence are limited, and further, it is common that diagnostics have good temporal resolution but not spatial resolution. The imaging diagnostic is strongly desired.

In plasma diagnostics, imaging techniques by multidirectional or stereoscopic observations including tomography have been developed. However, the freedom of sightline and detector setting is often limited in the practical plasma experiment. Therefore, as a next step, we focus on the mathematics of inverse problem for threedimensional (3D) image reconstruction with a single directional view. In this paper, we propose a technique of microwave holography with the planar arrangement of detectors. The feasibility is studied. After overviewing the prospect in the next section, modeling and formulation of observation are described in Sec. 3. A numerical method of reconstruction is discussed and examined in Secs. 4 and 5.

\section{Microwave Holography}

The laser holography utilizes the complex amplitude of light including power and phase information. In visible light digital holography, interference fringes of the refer-

author'se-mail: tsuchiya.hayato@nifs.ac.jp

*) This article is based on the presentation at the 27th International Toki Conference (ITC27) \& the 13th Asia Pacific Plasma Theory Conference (APPTC2018). ence light and the light from object are captured by a CCD camera, as shown in Fig. 1 (a). In microwave diagnostics, it is advantageous that the complex amplitude can be measured directly by mixing with the local waves as shown in Fig. 1 (b). Laser holography is well known and is utilized in various fields. However, in the plasma field, it has not yet been introduced. Moreover, in the frequency band where the microwave strongly interacts with plasma, the simultaneous imaging device with good resolution is not common.

For the microwave imaging reflectometry (MIR) and the electron cyclotron emission imaging (ECEI), our group has developed the Horn-antenna Millimeter-wave Imaging Device (HMID) [1] and the Local oscillator Integrated an-

(a) Visible Laser

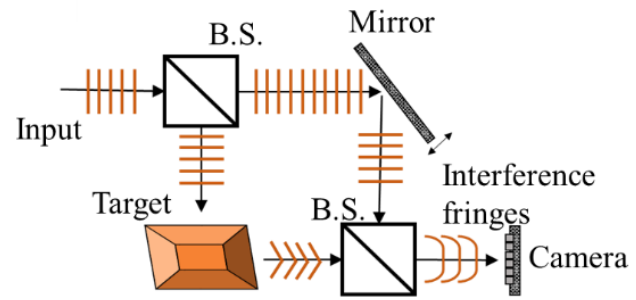

(b) Microwave

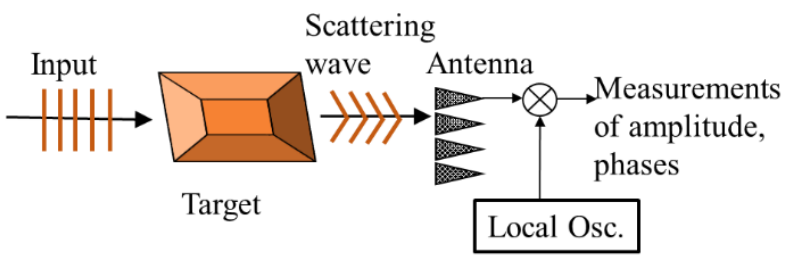

Fig. 1 Comparison between holography systems using (a) visible light (laser) and (b) microwave. 


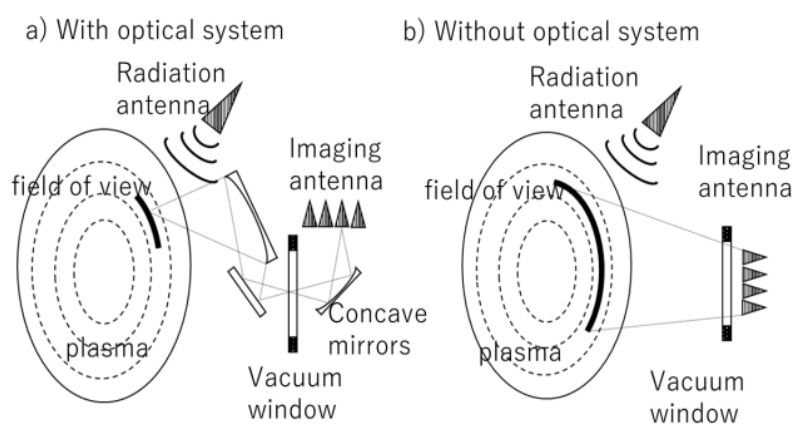

Fig. 2 The concept of imaging a) with optical system and b) without optical system.

tenna Array (LIA) [2], both of which possess horn antennas in planar arrangement, and they are applied to LHD experiments [3]. In such applications, the imaging devices were used together with optical systems which consisted of lens or mirrors. In this case, as shown in Fig. 2, in order to focus the microwave beam, the view area is a part of the side surface of the toroidal plasma. In contrast, without an optics system, a wide field of view would be secured. Known examples of no-lens optics system are the lensless camera of visible light and the synthetic aperture radar (SAR) of millimeter wave. In plasma microwave diagnostics, the number of detector points would be much fewer than that of CCD camera of visible light, and the wide scan by moving either the detector or the objects, such as SAR, is not feasible for transitional plasma. To obtain a $3 \mathrm{D}$ image from a limited number of detectors and sightlines, the inverse problem in a frame of ill-conditioned equation must be examined for microwave holography.

\section{Model of Microwave Propagation}

The microwave propagation is modeled in such a simple scheme as shown in Fig. 3. This diagnostic system model is applicable to both reflectometry and scattering measurement. The microwave (RF) is radiated from the source whose position is denoted as $\boldsymbol{p}_{\mathrm{s}}=\left(x_{0}, y_{0}, z_{0}\right)$. A stimulated particle in the target emits a spherical microwave. In the superposition of individual emissions, the scattering rate in the target is assumed to distribute as $f\left(\boldsymbol{p}_{\mathrm{t}}\right)$, which is related to the volume profile of electron density in scattering with the Born approximation and to the reflection surface profile when the density of target plasma is larger than the cutoff density. The scattering wave that is originated from an element of target located at $\boldsymbol{p}_{\mathrm{t}}=(x, y, z)$ reaches a detector located at $\boldsymbol{p}_{\mathrm{d}}=\left(x^{\prime}, y^{\prime}, z^{\prime}\right)$. The elemental wave $s_{\text {std }}$, which propagates via $\boldsymbol{p}_{\mathrm{s}}, \boldsymbol{p}_{\mathrm{t}}$ and $\boldsymbol{p}_{\mathrm{d}}$, is expressed as follows:

$$
s_{\text {std }}=S_{\mathrm{T}}\left(\boldsymbol{p}_{\mathrm{s}}, \boldsymbol{p}_{\mathrm{t}}\right) f\left(\boldsymbol{p}_{\mathrm{t}}\right) S_{\mathrm{R}}\left(\boldsymbol{p}_{\mathrm{t}}, \boldsymbol{p}_{\mathrm{d}}\right),
$$

where $S_{\mathrm{T}, \mathrm{R}}\left(\boldsymbol{p}_{1}, \boldsymbol{p}_{2}\right)$ is a function that represents the wave propagation from $\boldsymbol{p}_{1}$ to $\boldsymbol{p}_{2}$; the $S_{\mathrm{T}}$ depends on the model of the transmitted wave, for example, such as a spherical

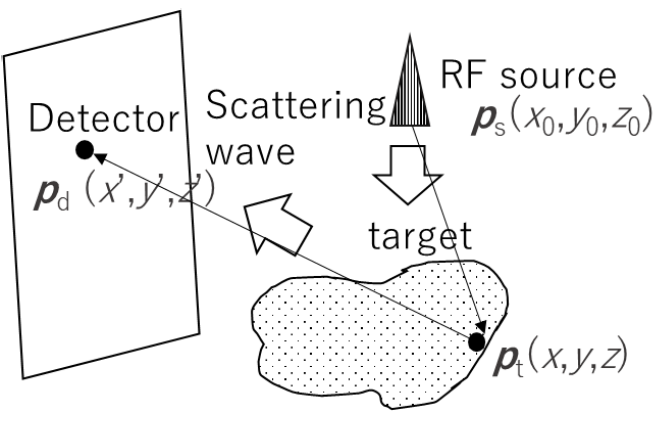

Fig. 3 Model of microwave holography.

wave or a plane wave. When one takes a model of spherical wave also for transmission, one has $S_{\mathrm{T}, \mathrm{R}}\left(\boldsymbol{p}_{1}, \boldsymbol{p}_{2}\right)=$ $\exp \left(-i k r_{12}\right) /\left\|r_{12}\right\|$ for the wavenumber $k$ and $r_{12}=\left\|\boldsymbol{p}_{1}-\boldsymbol{p}_{2}\right\|$. The Gaussian beam expression might be more appropriate to express the incident wave injected from the RF source point. The actual measurement $g\left(\boldsymbol{p}_{\mathrm{d}}\right)$ is the superposition of waves coming from all points of the target. Thereby, $g\left(\boldsymbol{p}_{\mathrm{d}}\right)$ is expressed in the form of integral:

$$
g\left(\boldsymbol{p}_{\mathrm{d}}\right)=\int_{V} s_{\mathrm{std}} d \boldsymbol{p}_{\mathrm{t}}=\int_{V} h_{\mathrm{td}} f\left(\boldsymbol{p}_{\mathrm{t}}\right) d \boldsymbol{p}_{\mathrm{t}}
$$

where $h_{\mathrm{td}}$ is a function which depends on the geometry of the source and the detector for the target.

By discretizing the integration, the above equation is approximated as

$$
g_{m}=\Sigma_{n=1}^{N} h_{m n} f_{n} \quad(m=1,2, \ldots, M),
$$

where we have $h_{m n}=h_{\mathrm{td}, m n} d \boldsymbol{p}_{\mathrm{t}}$, the number of detectors $M$ and that of target voxels $N$. Then, Eq. 3 is written simply as

$$
H \boldsymbol{f}=\boldsymbol{g},
$$

where $H$ is a matrix with elements $h_{m n}$; and $\boldsymbol{p}$ and $\boldsymbol{g}$ are column vectors. Since $H$ is derived from the product of wave propagation functions, it is generally a complex matrix. The $\boldsymbol{g}$ has also complex components related to the measurement values of power and phase, while $f$ can be assumed a real vector so long as the wave scatters without phase shift. In holographic image reconstruction, Eq. 4 is a system of linear equations to be solved for the unknown $\boldsymbol{f}$. A planar array of microwave receivers for 3D target suggests an inverse problem to be solved under the condition of measurements weakly independent and few as $M \ll N$.

\section{Reconstruction from Complex Amplitudes}

In regard to Eq.4, which will be underdetermined in our microwave holography, the least squares method of $\|H \boldsymbol{f}-\boldsymbol{g}\|^{2} / M \rightarrow$ min leads to the normal equation $\left(H^{*} H\right) \boldsymbol{f}=H^{*} \boldsymbol{g}$, where $H^{*}$ is the Hermitian transpose of $H$. The coefficient matrix $H^{*} H$ is rank deficient whenever $M<N$ and may be ill-conditioned in general. In 
proceeding to the Tikhonov regularization, the constrained minimization can be so extended as to use plural terms of regularization, that is, the Lagrangian function of the form

$$
\frac{1}{M}\|H \boldsymbol{f}-\boldsymbol{g}\|^{2}+\sum_{j} \gamma_{j}\left\|C_{j} \boldsymbol{f}\right\|^{2} \rightarrow \min ,
$$

with sets of multiplier $\gamma_{j}(>0)$ and square matrix $C_{j}$ [5]. In the present case study, we use two terms with $C_{1}$ and $C_{2}$, which are chosen to be the 3D Laplacian operator and the identity matrix, respectively. As well-known in plasma diagnostics, the Laplacian operator is effective to let the image value change smoothly at neighboring voxels. Meanwhile, the identity matrix is given the role of keeping the following normal equation well-conditioned.

Applying the Cholesky decomposition to the regularization terms leads to an extended normal equation written as

$$
\left(\gamma_{1} R^{*} R+H^{*} H\right) \boldsymbol{f}=H^{*} \boldsymbol{g} .
$$

Here $R$ is such an upper triangular matrix that we have $\gamma_{1} R^{*} R=\gamma_{1} C_{1}{ }^{*} C_{1}+\gamma_{2} C_{2}{ }^{*} C_{2}$. Then, the solution of Eq. 6 can be written in the ordinary form of series expansion using the singular value decomposition (SVD) of $H R^{-1}=$ $U \Sigma V^{*}$. That is, we have

$$
\begin{aligned}
\hat{\boldsymbol{f}}\left(\gamma_{1}, \gamma_{2}\right) & =R^{-1} V\left(\gamma_{1} I+\Sigma^{*} \Sigma\right)^{-1} \Sigma^{*} U^{*} g \\
& =\Sigma_{j=1}^{M} w_{j} a_{j} R^{-1} \boldsymbol{v}_{j},
\end{aligned}
$$

with the Tikhonov window and the coefficients

$$
w_{j}=\frac{1}{1+\frac{\gamma_{1}}{\sigma_{j}^{2}}}, \quad a_{j}=\frac{\boldsymbol{u}_{j}^{*} \boldsymbol{g}}{\sigma_{j}},
$$

for $M \leqq N$, where $\boldsymbol{u}_{j}$ and $\boldsymbol{v}_{j}$ are the orthonormal column vectors of $U$ and $V$, and the singular values $\sigma_{m}$ are diagonal elements of $\Sigma$. The generalized cross-validation (GCV) in its original expression [4] can be used for optimizing the value of $\gamma_{1}$ with respect to the term $R^{*} R$, which includes the ratio $\gamma_{2} / \gamma_{1}$ implicitly. The extension of the linear regularization of Tikhonov to the complex form is now available.

It is noted that the solution $\hat{f}$ is a complex vector in general even though it should be a real vector in our case. With inadequate measurements, the solution cannot be prevented from the appearance of the imaginary part. In Fig. 4, instructive results of a numerical test are exhibited. With respect to a complex matrix $H$ composed with random numbers, a simple target $f_{n}=\sin (2 \pi n / N)+i 0$ $(1 \leqq n \leqq N)$ was recovered from the projection $H \boldsymbol{f}$ without and with additive noise. It is seen that the target is well recovered in the no-noise case. However, the imaginary part error of $\hat{f}$ increases remarkably with the noise amplitude, and even becomes comparable with the real part in magnitude. Both solutions $\hat{f}$ were obtained with agreement good enough to the data $\boldsymbol{g}$, that is, with small magnitudes of the residuals.

A wise and effective countermeasure is to divide the entries of $H$ and $\boldsymbol{g}$ to real and imaginary parts and define

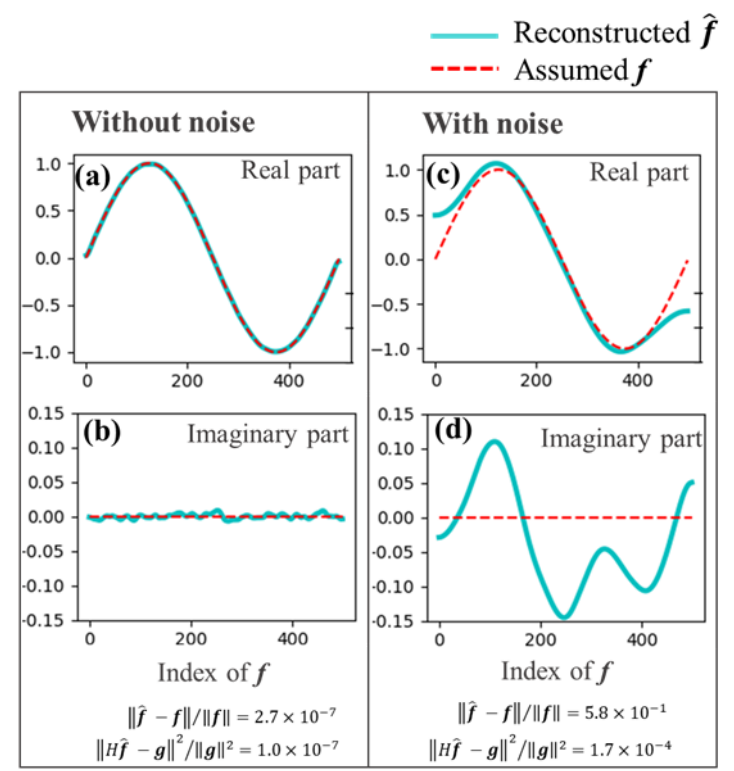

Fig. 4 Results of test inversion using Eq. 7 and GCV: Real and imaginary part of the solution $\hat{\boldsymbol{f}}$ from noiseless data (a) (b) and from noise corrupted data (c) - (d); $M=50$, $N=500$. In noisy case, uniform random numbers whose amplitude is as large as $50 \%$ of the average of $|H \boldsymbol{f}|_{m}$ were added to $\operatorname{Re}(H \boldsymbol{f})_{m}$ and $\operatorname{Im}(H \boldsymbol{f})_{m} ; S / N=4$.

an inverse problem of twice larger size in column. That is, for the same target $\boldsymbol{f}$, we have $H^{\prime} \boldsymbol{f}=\boldsymbol{g}^{\prime}$ with

$$
\begin{aligned}
& H^{\prime}=\left[\begin{array}{ccc}
\operatorname{Re}\left(h_{11}\right) & \ldots & \operatorname{Re}\left(h_{1 N}\right) \\
\vdots & \operatorname{Re}\left(h_{m n}\right) & \vdots \\
\operatorname{Re}\left(h_{M 1}\right) & \ldots & \operatorname{Re}\left(h_{M N}\right) \\
\operatorname{Im}\left(h_{11}\right) & \ldots & \operatorname{Im}\left(h_{1 N}\right) \\
\vdots & \operatorname{Im}\left(h_{m n}\right) & \vdots \\
\operatorname{Im}\left(h_{M 1}\right) & \ldots & \operatorname{Im}\left(h_{M N}\right)
\end{array}\right] \\
& \boldsymbol{g}^{\prime}=\left[\begin{array}{llllll}
\operatorname{Re}\left(g_{1}\right) & \cdots & \operatorname{Re}\left(g_{M}\right) & \operatorname{Im}\left(g_{1}\right) & \cdots & \operatorname{Im}\left(g_{M}\right)
\end{array}\right]^{T} .
\end{aligned}
$$

With this definition of real-value problem, the solution $\hat{\boldsymbol{f}}$ is formally the same with Eqs. 7 and 8, where the Hermitian transpose * is replaced by the simple transpose, and the number of terms is replaced by $M^{\prime}=2 M$. The twice-larger size of $\boldsymbol{g}^{\prime}$ looks reasonable because the acquisition of complex amplitude requires two independent measurements of power and phase.

\section{Effect of Detector Arrangement}

Now, the above formulation of Tikhonov inversion is examined on the back scattering with the Born approximation. Toward the target whose center position $\boldsymbol{p}_{\mathrm{t} \text {,center }}=$ $(0,0,0)$ as shown in Fig. 5 (a), the RF source located at $\boldsymbol{p}_{\mathrm{s}}$ $=(0,0,-100 \mathrm{~mm})$ injects a $30 \mathrm{GHz}$ spherical wave upwards in $z$ direction. The scattered wave is received by a 


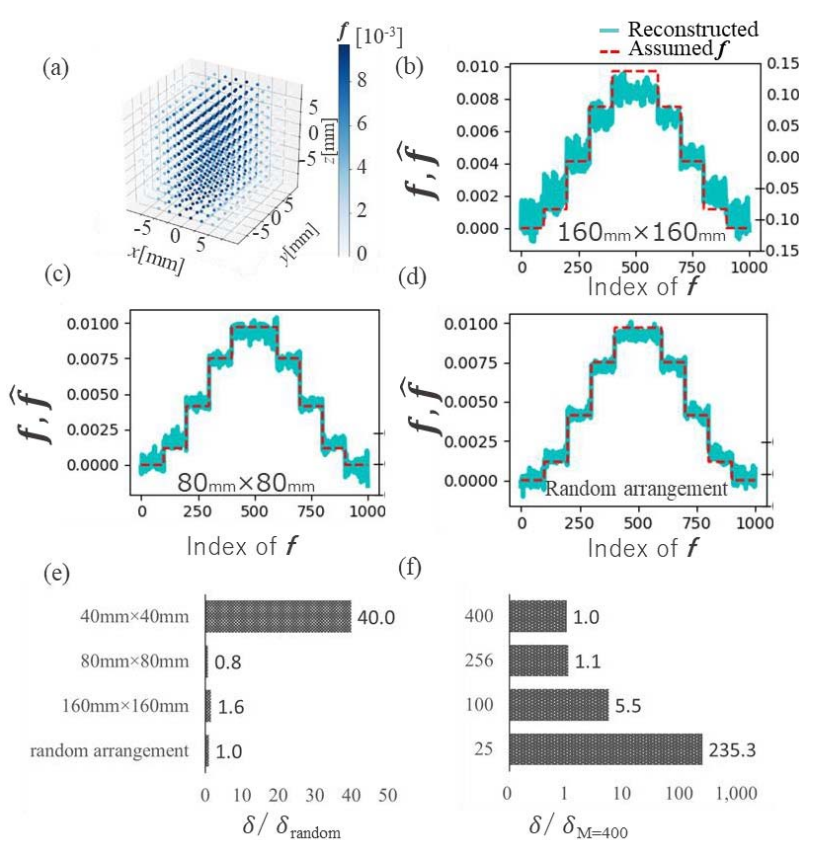

Fig. 5 Numerical test with different arrangements of detector. (a) Assumed scattering rate profile $f\left(\boldsymbol{p}_{\mathrm{t}}\right)=0.01 \cos ^{2}$ $(\pi x / 100)$ over the area of $-50 \mathrm{~mm}<x, y, z<50 \mathrm{~mm}$ (equally divided as $N=10 \times 10 \times 10=1000$ ); (b), (c) the images $\hat{f}$ in component row for the array stretches of $160 \mathrm{~mm} \times 160 \mathrm{~mm}$ and $80 \mathrm{~mm} \times 80 \mathrm{~mm}$ with $M=10 \times$ $10=100$; (d) $\hat{f}$ for a set of randomly located detectors of $M=100$; (e), (f) Change of $\delta$ with the array stretch $(M=100)$ and with the number of detectors $M(80 \mathrm{~mm}$ $\times 80 \mathrm{~mm}$ stretch).

planar array, which is set in the $x y$ plane of $z=-100 \mathrm{~mm}$ with the detectors that are arranged in tetragonal lattice. Using the same noise addition condition as that in Fig. 4 in data generation, the results of image reconstruction are shown in Fig. 5 with the change of detector arrangement, the real values of $\hat{\boldsymbol{f}}$ being guaranteed. While the GCV is valid primarily for choosing the best value of $\gamma_{1}$, it was used empirically for searching the best set of $\left(\gamma_{1}, \gamma_{2}\right)$ [5].

In Fig. 5 , the effect of detector arrangement is clear. When the reconstruction error $\delta$ is evaluated as $\delta=\| \hat{\boldsymbol{f}}-$ $\boldsymbol{f} \|^{2} / N$, it is changed by one order of magnitude with the change of arrangement, that is, the size of planar array and the number of detectors. With the geometry of tetragonal lattice such as HMID, a good reconstruction is obtained for the detector plane of $80 \mathrm{~mm} \times 80 \mathrm{~mm}$ wide as in Fig. 5 (c). However, a wider plane gives a worse reconstruction, as in Fig. 5 (b), since the detector sightline becomes similar in the edge region of the plane because the plane inclines against the target. The matrix $H^{\prime}$ is likely to lose the rank in numerically. This effect is explained by the quality of the matrix $H^{\prime}$, whose condition number $\kappa=\sigma_{\max } / \sigma_{\min }$ increases from $2.5 \times 10^{4}$ to $8.2 \times 10^{4}$. When the detectors are located randomly around the target, the reconstruction improves as shown in Fig. 5 (d), with a decreased value of $\kappa=4.4 \times 10^{3}$. The errors $\delta$ are summarized in Fig. 5 (e).

Another result is shown in Fig. 5 (f). For a fixed stretch of detector plane, the number of detectors $M$ in tetragonal lattice is changed. Larger $M$ gives better reconstruction as expected, whereas the manufacturing cost would be proportional to $M$.

\section{Summary}

The microwave holography for plasma diagnostic has been studied with the direct measurement of complex amplitude. Due to the microwave wavelength, the detector size is large and the number of detectors is much smaller than that of CCD picels. This situation leads to an illconditioned inverse problem. A solution for real-valued imaging objects has been examined numerically on the back-scattering observation. The results suggest that a good reconstruction is possible with the appropriate arrangement of planar detectors.

\section{Acknowledgments}

This work is performed with the support and under the auspices of the NIFS Collaboration Research program (NIFS17KLEP024, NIFS18KLEP029).

[1] Y. Nagayama et al., Rev. Sci. Instrum. 88, 044703 (2017).

[2] D. Kuwahara et al., J. Plasma Fusion Res. 8, 649 (2009).

[3] H. Tsuchiya et al., Plasma Fusion Res. 13, 3402063 (2018).

[4] G.H. Golub, M. Heath and G. Wahba, Technometrics 21, 215 (1979).

[5] T. Kobayashi et al., Rev. Sci. Instrum. 89, 123502 (2018). 